\title{
The benefits of public transport
}

Some $55 \%$ of the world's population currently lives in urban areas, and this number is expected to reach $70 \%$ by mid-century. This condensation of humanity into compact zones reflects fundamental causal factors. People can achieve more when working together, coordinating their diverse skills and knowledge. Of course, cities also bring efficiencies of energy use, water and food distribution and provision of the myriad other goods people need. They are probably unavoidable in any energy- and information-intensive civilization.

Our future cities, some believe, could be key sources of the ideas and behavioural change required to meet the environmental challenges of the future, especially avoiding catastrophic global warming. They present opportunities to reduce per capita energy consumption. Yet cities also present problems, such as traffic congestion, which wastes time and energy and creates additional $\mathrm{CO}_{2}$ emissions. Currently, some $20 \%$ of all $\mathrm{CO}_{2}$ emissions come from road traffic, and that may increase, as armies of delivery vehicles and self-driving cars take to the roads in the future.

Over the past two decades, statistical physicists have discovered a variety of structural and dynamic regularities of cities. As organic structures both shaped by and shaping human activities, these sprawling, irregularly shaped zones follow mathematical scaling laws. For cities spanning some five orders of magnitude in size, some quantities, such as the extent of physical infrastructure, scale sub-linearly with size, meaning the relative costs decrease with city size. In contrast, many quantities reflecting human interactions, such as economic activity, scale superlinearly - cities become even more productive with size.

But statistical physics can go further as well, in giving insight into how our cities might be improved, especially by helping to limit traffic congestion and associated fuel use and emissions. Oddly, prevailing ideas in transportation research suggest there's little to be done. Thinking of three decades holds that the character of traffic flows is largely determined only by the population density. Some economic analyses have even suggested that building more roads doesn't help, nor does more mass transit. New traffic always emerges to take the place of any free road space.
New research tells a very different story - that public transport is among the most direct ways to reduce congestion, if done in the right way. The key is making sure that the layout of a public transport system makes access to mass transit easy for a high fraction of people in any city.

To get a better picture of what influences traffic, physicists Vincent Verbavatz and Marc Barthelemy set out to build a simple schematic model capturing the simplest elements in the interplay between driving and public transport, while neglecting secondary details (PLoS ONE 14, e0219559; 2019). Their aim was to derive the basic relationship between two key variables first, the fraction of people in a city who choose to drive, rather than taking public transport, and second, the fraction of a city's population living quite close to public transport, and so having easy access to it.

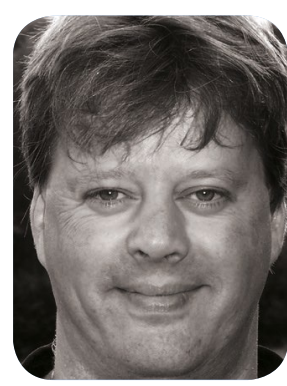

\section{Our future cities could be key sources of the ideas and behavioural change required to meet the environmental challenges of the future.}

The model requires quite a few assumptions, and setting parameters including the average driving velocity and speed of public transport, as well as the psychological value people put on avoiding one extra hour of sitting in traffic. Yet most of these details turn out not to affect one qualitative result that emerges from the model. If $p$ is the fraction of people living 'close' to public transport, $P$ is the population and $T$ is the fraction of people who drive rather than take public transport, the model gives a strikingly simple prediction: $T / P=1-p$. The fraction of people driving should decrease in direct proportion to the fraction of people with easy access to transport.

Verbavatz and Barthelemy were then able to test this prediction using data for 25 large metropolitan areas from Europe, America, Asia and Australia. The figures fall almost exactly on the straight-line prediction, apart from a small scatter. Across these cities, the fraction of people driving to work decreases in direct proportion to the availability of mass transit, as estimated in this case by the fraction of the population living within one kilometre of a transit station.

One might wonder: why did no one discover this before? Probably, Barthelemy told me, because the required data didn't exist. Making quantitative estimates to test the model required modern data sources including TomTom navigation data and average driving speeds estimated from Google maps. But also, scientists don't make observations at random. What researchers measure often reflects possibilities raised by theoretical ideas. The new model is the first to suggest this simple pattern as a possibility.

"Before," he said, "no one had a predictive model. Economists often look for correlations and perform econometric analysis but do not have a model that makes an analytical prediction."

The result fits perfectly with the intuition that making public transport easier should tend to reduce automobile traffic by drawing people toward public transit alternatives. An older idea that traffic loads in a city are primarily determined by population density, and nothing else, implied that city authorities could only try to bring in more people, and fit them into smaller zones. In contrast, this new work suggests that city authorities have many more options. The parameter $p$, reflecting ease of access, obviously wraps up a host of real-world factors that influence how hard it is for people to choose public transport. These include not only geographical proximity to transport stations, but also things such as the frequency of service, availability of up-to-date information, and access to local buses and other services able to take people over short distances to public transport stations. All are targets for making $p$ larger.

This is a good example of how surprising insight can come out of combining complex sources of data. It's Big Data doing good. It's not going to solve our traffic problems, but at least it points the way to the most intelligent way to reduce traffic congestion: make public transport more effective.

\section{Mark Buchanan}

Published online: 3 September 2019 https://doi.org/10.1038/s41567-019-0656-8 